\title{
СИНТЕЗ НИТРИДА НИОБИЯ В УСЛОВИЯХ ТЕПЛОВОГО ВЗРЫВА СМЕСЕЙ НАНОПОРОШКА АЛЮМИНИЯ С ПЕНТАОКСИДОМ НИОБИЯ
}

\author{
Чудинова Александра Олеговна',
} chudinova.1509@mail.ru

\author{
Ильин Александр Петрович ${ }^{1}$ \\ genchem@mail.ru
}

\section{Роот Людмила Олеговна', tolbanova@mail.ru}

\section{Мостовщиков Андрей Владимирович', avmost@tpu.ru}

\author{
Беспалова Екатерина Александровна², \\ katena.bespalova9570@gmail.com
}

\author{
Атулиа Манураджз, \\ atulyamanuraj@gmail.com \\ 1 Национальный исследовательский Томский политехнический университет, \\ Россия, 634050, г. Томск, пр. Ленина, 30. \\ ${ }^{2}$ Алтайский государственный технический университет им. И.И. Ползунова, \\ Россия, 659305, г. Бийск, ул. Имени Героя Советского Союза Трофимова, 27. \\ ${ }^{3}$ Индийский технологический институт, \\ Индия, г. Канпур, 208 016, У.П.
}

Актуальность. Получение тугоплавких нитридов в воздухе в условиях теплового взрыва смесей нанопорошка алюминия с оксидами металлов представляет практический интерес для материаловедения и для теории реакционной способности воздуха при высоких температурах. Такой синтез является наименее энергозатратным и не требует сложного оборудования. Для протекания синтеза необходим только нагрев исходной шихты, затем процесс протекает самопроизвольно. Синтез нитридсодержащих продуктов в воздухе с использованием атмосферного азота при нормальных условиях представляет интерес для получения новых видов керамики, добавок в обрабатывающий инструмент, для дисперсного упрочнения полимерных и композиционных материалов.

Цель исследования: экспериментально определить состав продуктов сгорания смесей нанопорошка алюминия с пентаоксидом ниобия в воздухе.

Объект: порошок, содержащий нитрид ниобия, полученный при сжигании смеси нанопорошка алюминия с пентаоксидом ниобия в воздухе.

Методы: рентгенофазовый анализ (дифрактометр Дифрей-401), дифференциальный термический анализ (термоанализатор SDT Q600, фирма Instrument). На основании результатов дифференциального термического анализа были рассчитаны четыре параметра активности смесей: температура начала окисления $\left(t_{\text {н.o, }}{ }^{\circ} \mathrm{C}\right)$, степень окисленности $(\alpha, \%)$, максимальная скорость окисления $\left(V_{\max }, \mathrm{Mr} / \mathrm{Mин}\right)$, удельный тепловой эффект $(\Delta H$, Дж/г). Рентгенофазовый анализ использовали для изучения фазового состава продуктов окисления.

Результаты. Процесс горения смесей нанопорошка алюминия с пентаоксидом ниобия в воздухе протекал в две стадии с формированием нитрида ниобия $\mathrm{Nb}_{2} \mathrm{~N}$. Согласно рентгенофазовому анализу, выход нитрида ниобия в продукте сгорания смеси НП Al: $\mathrm{Nb}_{2} \mathrm{O}_{5}=3: 1$ в мольном соотношении (при массе смесей $\mathrm{H}$ П Al: $\mathrm{Nb}_{2} \mathrm{O}_{5}=2,64: 1,36$ ) достигал максимума и составлял 47 отн. \%. Расчет изобарно-изотермического потенциала показал, что нитрид ниобия должен окисляться кислородом воздуха. Причиной стабилизации кристаллической фазы $\mathrm{Nb}_{2} \mathrm{~N}$ является дезактивация кислорода воздуха излучением горящего нанопорошка алюминия.

\section{Ключевые слова:}

Нитрид ниобия, тепловой взрыв, нанопорошок, алюминий, азот воздуха, рентгенофазовый анализ, дифференциальный анализ, нитрид алюминия, параметры активности.

\section{Введение}

Оксид ниобия $\left(\mathrm{Nb}_{2} \mathrm{O}_{5}\right)$ является продуктом первичной переработки минерального сырья в металлический ниобий и является полезным компонентом специальных сталей [1]. Рост промышленного потенциала РФ связан с глубокой переработкой минеральных ресурсов и, в частности, с получением нитридов ниобия. Ранее было экспериментально показано, что при сжигании смесей нанопорошка алюминия с оксидами титана, циркония и гаф- 
ния в воздухе в продуктах сгорания стабилизировались соответствующие нитриды с общей формулой $\mathrm{MeN}$ [2]. Для элементов V группы Периодической системы аналогичные эксперименты не проводились.

При окислении порошкообразного ниобия при нагревании в воздухе в продуктах окисления была обнаружена кристаллическая фаза нитрида ниобия [1]. Получение тугоплавких нитридов в воздухе в условиях теплового взрыва смесей нанопорошка алюминия с оксидами металлов представляет практический интерес для материаловедения и для теории реакционной способности воздуха при высоких температурах [3-7].

Процесс сжигания свободно насыпанной навески проводится с помощью инициирования горения при атмосферном давлении [8]. Такой синтез является наименее энергозатратным и не требует сложного оборудования. Для протекания синтеза необходим только нагрев исходной шихты, затем процесс протекает самопроизвольно $[9,10]$.

Целью настоящей работы является экспериментальное определение состава продуктов сгорания смесей нанопорошка алюминия с пентаоксидом ниобия в воздухе.

\section{Методики экспериментов и характеристики веществ}

В работе использовался нанопорошок (НП) алюминия, полученный с помощью электрического взрыва проводников [2]. Нанопорошок алюминия, полученный в среде аргона, является пирофорным, т. е. воспламеняется при контакте с воздухом. Его пассивировали медленным окислением малыми добавками воздуха [11-15]. Нанопорошок алюминия характеризовался следующими параметрами активности: температура начала окисления $400{ }^{\circ} \mathrm{C}$; степень окисленности 47,3\%; максимальная скорость окисления 0,19 мас. \%/ ${ }^{\circ} \mathrm{C}$; удельный тепловой эффект 8,8 кДж/г. В эксперименте использовали промышленный порошок пентаоксида ниобия с содержанием пентаоксида ниобия в порошке более чем 99 мас. \% со средним диаметром частиц 20-40 мкм.

Массы образцов подбирались таким образом, чтобы горение смесей было устойчивым с достаточным количеством продуктов сгорания для проведения последующих физико-химических анализов (табл. 1) [16-18].

C помощью дифференциального термического анализа (ДТА) диагностировали исходные смеси порошков ( $\mathrm{H \Pi} \mathrm{Al}, \mathrm{Nb}_{2} \mathrm{O}_{5}$ ). Это позволяет определить скорость окисления и степень превращения при определенных температурах.

Исходные смеси нанопорошка алюминия с пентаоксидом ниобия, используя термоанализатор STD Q600, проверяли на пирофорность путем расчета по ДТА четырех параметров активностей [3]: температура начала окисления $\left(t_{\text {н.o }},{ }^{\circ} \mathrm{C}\right)$, максимальная скорость окисления ( $v_{\max }$, мг/мин), степень превращения (степень окисленности) нанопорошка в определенном температурном интервале $(\alpha, \%)$, удельный тепловой эффект $(\Delta H / \Delta m$, кДж/г) [3]. Все приготовленные смеси имеют температуру начала окисления выше $300{ }^{\circ} \mathrm{C}$, поэтому они не пирофорны (табл. 2) и их приготовление не требует особых мер предосторожности.

Таблица 1. Состав исследуемых смесей НП АІ с пентаоксидом ниобия

Table 1. Composition of the investigated mixtures of NP Al with niobium pentoxide

\begin{tabular}{|c|c|c|c|c|}
\hline \multirow{2}{*}{ 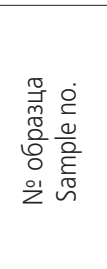 } & \multicolumn{2}{|c|}{$\begin{array}{c}\text { Состав образца, мас. части } \\
\text { Sample composition, } \\
\text { wt. parts }\end{array}$} & \multirow{2}{*}{$\begin{array}{c}\text { Соотноше- } \\
\text { ние } \mathrm{H} П \\
\mathrm{Al}: \mathrm{Nb}_{2} \mathrm{O}_{5} \\
\text { мольное } \\
\text { Ratio NP } \\
\begin{array}{c}\mathrm{Al}: \mathrm{Nb}_{2} \mathrm{O}_{5} \\
\text { molar }\end{array}\end{array}$} & \multirow{2}{*}{$\begin{array}{c}\text { Массовая } \\
\text { доля НП Al } \\
\text { в смеси, \% } \\
\text { Mass frac- } \\
\text { tion of NP Al } \\
\text { in the } \\
\text { mixture, \% }\end{array}$} \\
\hline & $\begin{array}{l}\text { Macca HП } \\
\text { Al, } r \\
\text { Weight of } \\
\text { NP Al, g }\end{array}$ & $\begin{array}{l}\text { Масса порош- } \\
\text { ка } \mathrm{Nb}_{2} \mathrm{O}_{5},\ulcorner \\
\text { Weight of pow- } \\
\text { der } \mathrm{NP} \mathrm{Nb}_{2} \mathrm{O}_{5}, \mathrm{~g}\end{array}$ & & \\
\hline 1 & 1,60 & 2,40 & $2: 1$ & 40 \\
\hline 2 & 2,06 & 1,94 & $3: 1$ & 51 \\
\hline 3 & 2,64 & 1,36 & $4: 1$ & 66 \\
\hline 4 & 2,80 & 1,20 & $5: 1$ & 70 \\
\hline $5-\mathrm{NPAl}$ & 0,00 & 4,00 & - & 100 \\
\hline
\end{tabular}

Рентгенофазовый анализ осуществляли методом порошка, используя дифрактрометр «Дифрей-401» с анодом из железа. Длина волны излучения анода $\lambda\left(\mathrm{Fe}_{\mathrm{K}_{\alpha}}\right)=0,193 \mathrm{Hм}[4]$.

Обработку рентгенограмм проводили по стандартному методу сравнения рефлексов рентгенограмм исследуемых образцов с рентгенограммами из базы данных картотеки ASTM (Американское общество испытаний материалов). Необходимым минимальным критерием отбора присутствующих фаз являлось совпадение минимум с тремя наиболее интенсивными рефлексами эталона.

\section{Результаты экспериментов}

На основе данных дифференциального термического анализа была оценена реакционная способность исходного нанопорошка алюминия и исследуемых смесей. Типичная термограмма представлена на рис. 1.

Были записаны термограммы, и рассчитаны четыре параметра активности исследуемых смесей [13-21]. Благодаря методу дифференциального термического анализа, были рассчитано четыре параметра химической активности, позволяющие характеризовать свойства нанопорошка (табл. 2).

С ростом содержания нанопорошка алюминия наблюдалось повышение температуры начала окисления $\left(T_{\text {н.o. }}\right)$, исследуемых смесей нанопорошка алюминия с пентаоксидом ниобия (табл. 2), с 310 до 410 ㄷ. При этом происходило увеличение степени окисленности с 26,06 до 40,69 \% и максимальной скорости окисления с 0,03 до 0,18 мас. \% . Также при анализе полученных данных было установлено закономерное увеличение удельного теплового эффекта смесей с 2960,06 до 5238,12 Дж/г при увеличении содержания нанопорошка алюминия в смесях. 


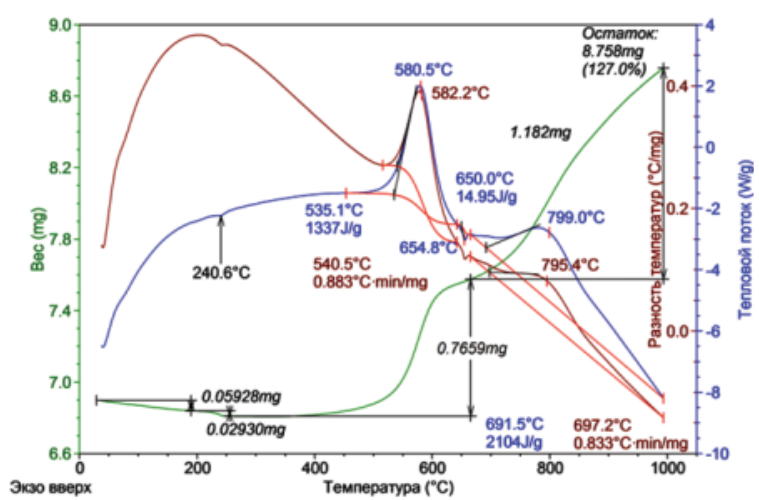

Pис. 1. Термограмма смеси нанопорошка алюминия с порошком пентаоксида ниобия (атмосфера - воздух скорость нагрева $10{ }^{\circ} \mathrm{C} / \mathrm{Mин,} \mathrm{масса} \mathrm{навески} \mathrm{-}$ 8,758 Mг, $\left.\mathrm{H} \Pi \mathrm{Al}: \mathrm{Nb}_{2} \mathrm{O}_{5}=3: 1\right)$

Fig. 1. Thermogram of aluminum nanopowder and niobium pentaoxide mixture (air, heating rate $10^{\circ} \mathrm{C} / \mathrm{min}$, sample weight is 8,758 mg, NP Al: $\mathrm{Nb}_{2} \mathrm{O}_{5}=3: 1$ )

Таблица 2. Параметры активности смесей нанопорошка алюминия с пентаоксидом ниобия

Table 2. Parameters of niobium pentaoxide and aluminum nanopowder mixtures activity

\begin{tabular}{|c|c|c|c|c|}
\hline $\begin{array}{l}\text { № образца } \\
\text { Sample no. }\end{array}$ & $T_{\text {H.o }} / T_{\text {n.o. }},{ }^{\circ} \mathrm{C}$ & $\alpha, \%$ & $\begin{array}{c}V_{\max }, \text { Mac. } \% / c \\
V_{\max }, \text { wt. \%/s }\end{array}$ & $\begin{array}{c}\Delta H, \text { кДж/r Al } \\
\Delta H, \mathrm{~kJ} / \mathrm{g} \mathrm{Al}\end{array}$ \\
\hline 1 & 310 & 26,06 & 0,03 & 2,9 \\
\hline 2 & 400 & 31,64 & 0,08 & 3,4 \\
\hline 3 & 330 & 46,50 & 0,19 & 6,1 \\
\hline 4 & 410 & 40,69 & 0,18 & 5,2 \\
\hline 5 & 400 & 47,3 & 0,19 & 8,8 \\
\hline
\end{tabular}

На рис. 2 представлена рентгенограмма продуктов сгорания образца № 2 (табл. 1). Данные для определения кристаллических фаз были взяты из международной картотеки ASTM. При увеличении содержания $\mathrm{H \Pi} \mathrm{Al}$ в продуктах сгорания присутствуют фазы $\mathrm{Nb}_{2} \mathrm{~N}$ и $\mathrm{Al}_{2} \mathrm{O}_{3}$ (рис. 2). На рентгенограмме рефлекс интенсивностью $100 \%$ соответствует рефлексу интенсивностью $100 \%$ фазы нитрида ниобия (рис. 2) (международная картотека PDF № 391398).

Исследованы продукты сгорания в жидком азоте в смесях компактированных образцов нанопорошка алюминия с пентаоксидом ниобия. Газопроницаемость образцов (масса каждого образца была 3 г) при гетерогенном горении систем «металл-газ» имеет большое значение. Давление компактирования влияет на величину пористости образца. Поэтому в дальнейшем образцы компактированы небольшим усилием $P=25$ кПа.

За счёт высокотемпературного связывания азота воздуха в газовой фазе и последовательного вытеснения кислорода азотом из $\gamma$-оксида алюминия происходит процесс формирования нитрида алю- миния. Эти результаты не противоречат ранее предложенному механизму горения нанопорошка алюминия в воздухе [22, 23]. Одновременно формирование фазы AlN происходит путем прямого взаимодействия алюминия с азотом.

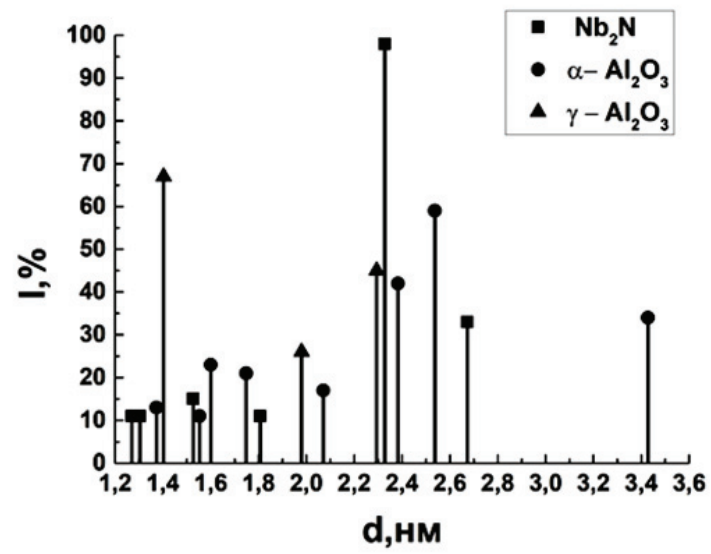

Pис. 2. Рентгенограмма продуктов сгорания в воздухе смеси нанопорошка алюминия с пентаоксидом ниобия (массовое соотношение $\mathrm{H}$ П Al: $\mathrm{Nb}_{2} \mathrm{O}_{5}=3: 1$ )

Fig. 2. X-ray diagram of combustion products of niobium pentaoxide and aluminum nanopowder mixture (NP Al: $\mathrm{Nb}_{2} \mathrm{O}_{5}=3: 1$ )

\section{Заключение}

Предложен способ синтеза тугоплавкого нитрида ниобия $\mathrm{Nb}_{2} \mathrm{~N}$ сжиганием смесей нанопорошка алюминия с пентаоксидом ниобия $\mathrm{Nb}_{2} \mathrm{O}_{5}$. Процесс сжигания проводится с помощью инициирования горения свободно насыпанной навески при атмосферном давлении.

1. Формирование нитрида ниобия в присутствии кислорода происходит в условиях теплового взрыва, при котором происходит нетепловой процесс дезактивации триплетного кислорода путем его перевода в неактивное синглетное состояние [24].

2. Вероятно, что $\mathrm{Nb}_{2} \mathrm{~N}$ является наиболее термически устойчивым нитридом среди соединений ниобия с азотом.

\section{Выводы}

1. Максимальный выход нитрида ниобия $\mathrm{Nb}_{2} \mathrm{~N}$ в продуктах сгорания смесей нанопорошка алюминия с пентаоксидом ниобия в воздухе харак-

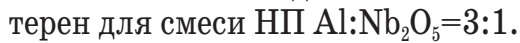

2. При горении в воздухе смесей нанопорошка алюминия с пентаоксидом ниобия (массовое со-

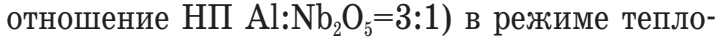
вого взрыва в составе продуктов сгорания содержание фазы нитрида ниобия достигает 47 отн. $\%$.

Работа выполнена при поддержке Государственного задания «Наука», проект № 11.1928.2017/4.6. 


\section{СПИСОК ЛИТЕРАТУРЫ}

1. Nobuzo Terao. Structure des Nitrures de Niobium // Japanese Journal of Applied Physics. - 1965. - V. 64. - № 5. - P. 353-367.

2. Назаренко О.Б., Ильин А.П., Тихонов Д.В. Электрический взрыв проводников. Получение нанопорошков металлов и тугоплавких неметаллических соединений. - Saarbrucken: LAP LAMBERT Academic Publishing GmbH\&Co/KG, 2012. - 274 c.

3. Уэндландт У. Термические методы анализа. - М.: Мир, 1978. $218 \mathrm{c.}$

4. Ковба Л.М., Трунов В.К. Рентгенофазовый анализ. - М.: МГУ, 1976. -232 c.

5. Тот Л. Карбиды и нитриды переходных металлов / пер. с англ. - М.: Мир, 1974. - 294 с.

6. Химическая технология ниобия и тантала / А.А. Маслов, Р.В. Оствальд, В.В. Шагалов, Е.С. Маслова, Ю.С. Горенюк. Томск: Изд-во Томского политехнического университета, 2010. -97 c.

7. Ниобий и тантал / А.Н. Зеликман, Б.Г. Коршунов, А.В. Елютин и др. - М.: Металлургия, 1990. - 295 с.

8. Самсонов Г.В. Нитриды. - Киев: Наукова думка, 1969. - 377 с.

9. Лорян В.Э., Боровинская И.П. 0 горении алюминия в азоте // Физика горения и взрыва. -2003 . - Т. 39. - № 5. - С. 45-54.

10. Физика и химия горения нанопорошков металлов в азотсодержащих газовых средах / под ред. А.А. Громова. - Томск: Издво Том. ун-та, 2007. - 322 с.

11. Шевченко В.Г., Кононенко В.И., Булатов М.А. 0 механизме окисления порошкообразных металлов в процессе их нагревания на воздухе // Физика горения и взрыва. - 1998. - № 1. C. $45-49$.

12. Химия синтеза сжиганием / под ред. М. Коидзуми / пер. с японск. - М.: Мир, 1998. -247 с.

13. Nico C., Monteiro T., Graça M.P.F. Niobium oxides and niobates physical properties: review and prospects // Progress in Materials Science. - 2016. - V. 80. - P. 1-37.

14. Zakorzhevsky V.V. Aluminum Nitride // Concise Encyclopedia of Self-Propagating High-Temperature Synthesis History, Theory, Technology, and Products. - Elsevier Science, 2017. - P. 16-18.
15. Nitrogen transfer properties in tantalum nitride based materials / S. Laassiri, C.D. Zeinalipour-Yazdi, C.R.A. Catlow, J.S.J. Hargreaves // Catalysis Today. - 15 May 2017. - V. 286. P. $147-154$.

16. Facile synthesis of powder-based processing of porous aluminum nitride / Kyungju Nam, Kicheol Hong, Hyeji Park, Heeman Choe // Journal of the European Ceramic Society. - April 2018. V. 38. - Iss. 4. - P. 1164-1169.

17. Ognjanovic S.M., Winterer M. Optimizing particle characteristics of nanocrystalline aluminum nitride // Powder Technology. - 15 February 2018. - V. 326. - P. 488-497.

18. Yamane H., DiSalvo F.J. Sodium flux synthesis of nitrides // Progress in Solid State Chemistry. - September 2018. - V. 51. P. 27-40.

19. On the synthesis of lithium boron nitride $\left(\mathrm{Li}_{3} \mathrm{BN}_{2}\right) / \mathrm{K}$. Sahni, M. Ashuri, S. Emani, J.A. Kaduk, K. Németh, L.L. Shaw // Ceramics International. - May 2018. - V. 44. - Iss. 7. - P. 7734-7740.

20. Synthesis and electrocatalytic performance of spherical core-shell tantalum (oxy) nitride nitrided carbon composites in the oxygen reduction reaction / M. Wassner, M. Eckardt, C. Gebauer, G.R. Bourret, N. Hüsing, R.J. Behm // Electrochimica Acta. 10 February 2017. - V. 227. - P. 367-381.

21. Activated reaction synthesis of silicon oxynitride from silica and silicon nitride / J. Fruhstorfer, F. Kerber, Ch. Weigelt, K. Moritz, Ch.G. Aneziris // Ceramics International. - May 2018. V. 44. - Iss. 7. - P. 8467-8475.

22. Low-temperature synthesis of tantalum carbide by facile one-pot reaction / Jinming Jiang, Song Wang, Wei Li, Zhaohui Chen // Ceramics International. - 2016. - V. 42. - № 6. - P. 7118-7124.

23. Горение нанопорошков металлов / А.А. Громов, Т.А. Хабас, А.П. Ильин и др. / под. ред. А.А. Громова. - Томск: Дельтоплан, 2008. - 382 c.

24. Schweiter C., Schmidt R. Physical Mechanisms of Generation and Deactivation of Singlet Oxygen // Chemical Review. - 2003. V. 103 (5). - P. 1685-1787.

Поступила 18.06.2018 г.

\section{Информация об авторах}

Чудинова A.O., аспирант отделения естественных наук Школа базового инженерного образования Национального исследовательского Томского политехнического университета.

Ильин А.П., доктор физико-математических наук, профессор отделения естественных наук Школы базового инженерного образования Национального исследовательского Томского политехнического университета.

Poom Л.O., кандидат технических наук, доцент отделения естественных наук Школы базового инженерного образования Национального исследовательского Томского политехнического университета.

Мостовщиков A.B., кандидат технических наук, научный сотрудник научно-исследовательской лаборатории СВЧ-технологий Инженерной школы ядерных технологий Национального исследовательского Томского политехнического университета.

Беспалова E.A., студентка Бийского технологического института (филиала) Алтайского государственного технического университета им. И.И. Ползунова.

Манурадж $A$., студент Индийского технологического института. 
UDC 544.45.452.2

\title{
SYNTHESIS OF NIOBIUM NITRIDE IN CONDITIONS OF HEAT EXPLOSION OF ALUMINUM NANOPOWDER AND NIOBIUM PENTOXIDE MIXTURES
}

\author{
Alexandra 0. Chudinova', \\ chudinova.1509@mail.ru
}

Alexander P. Ilyin',
genchem@mail.ru
Lyudmila 0. Root',
tolbanova@mail.ru

Andrey V. Mostovshchikov', avmost@tpu.ru

Ekaterina A. Bespalova²,

katena.bespalova9570@gmail.com

Atulya Manuraj ${ }^{3}$,

atulyamanuraj@gmail.com

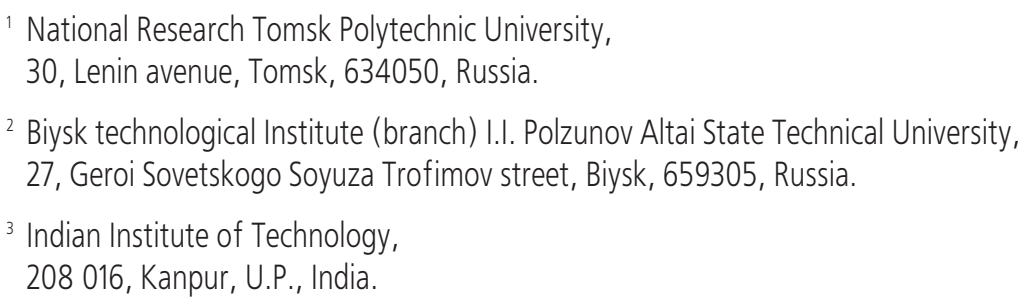

The relevance. The preparation of refractory nitrides in the air under the conditions of thermal explosion of aluminum nanopowder mixtures with metal oxides presents practical interest both for materials science and for the theory of the reactivity of air at high temperatures. This synthesis is the least energy-intensive, and it does not require complicated equipment. Only the heating of the initial charge is necessary for synthesis, then the process proceeds spontaneously.

The main aim of the research is to determine experimentally the composition of combustion products of aluminum nanopowder mixtures with niobium pentaoxide in the air, to substantiate theoretically the stabilization of niobium nitride $\mathrm{Nb}_{2} \mathrm{~N}$ in the air.

Object: powder containing niobium nitride obtained by burning a mixture of aluminum nanopowder with niobium pentoxide in the air. Methods: $x$-ray analysis (diffractometer Difrey-401), differential thermal analysis SDT Q600 Instrument company. On the basis of the results of the differential thermal analysis the authors have calculated four parameters of the mixtures activity: temperature of oxidation beginning $\left(t_{\text {st.ox, }}{ }^{\circ} \mathrm{C}\right)$, oxidation degree $(\alpha, \%)$, maximal oxidation speed $\left(v_{\max }, \mathrm{mg} / \mathrm{min}\right)$, specific thermal effect $(\Delta H, J / g) . X$-ray analysis was used for investigating crystal structure of oxidation ending products.

Results. Combustion of mixtures of aluminum nanopowder with niobium pentaoxide in the air proceeded in two stages with formation of

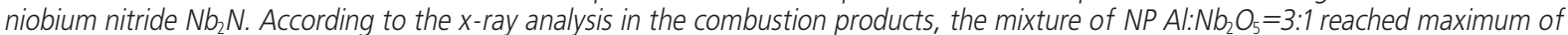
$47 \mathrm{rel}$. \%. The calculation of the isobaric-isothermal potential showed that niobium nitride should be oxidized by air oxygen. The reason of stabilization of $\mathrm{Nb}_{2} \mathrm{~N}$ crystalline phase is the air oxygen deactivation by emission of burning aluminum nanopowder.

\section{Key words}

Niobium nitride, thermal explosion, nanopowder, aluminum, air nitrogen, $X$-ray analysis, differential thermal analysis, aluminum nitride, parameters of activity.

The research was supported by the State Task «Nauka», project no. 11.1928.2017/4.6.

\section{REFERENCES}

1. Nobuzo Terao. Structure des Nitrures de Niobium [Structure of niobium nitride]. Japanese Journal of Applied Physics, 1965, vol. 64 , no. 5, pp. 353-367.

2. Nazarenko 0.B., Ilin A.P., Tikhonov D.V. Elektricheskiy vzryv provodnikov. Poluchenie nanoporoshkov metallov $i$ tugoplavkikh nemetallicheskikh soedineniy [Wire electrical explosion. Metal and refractory nonmetal compounds nanopowders production].
Saarbrucken, LAP LAMBERT Academic Publishing $\mathrm{GmbH \& Co/KG,} \mathrm{2012.274} \mathrm{p}$

3. Uendlandt U. Termicheskie metody analiza [Thermal analysis methods]. Moscow, Mir Publ., 1978. 218 p.

4. Kovba L.M., Trunov V.K. Rentgenofazovy analiz [X-ray diffraction analysis]. Moscow, MGU Publ., 1976. 232 p.

5. Tot L. Karbidy i nitridy perekhodnykh metallov [Carbides and nitrides of transition metals]. Translated from English. Moscow, Mir Publ., 1974. 294 p. 
6. Maslov A.A., Ostvald R.V., Shagalov V.V., Maslova E.S., Gorenyuk Yu.S. Khimicheskaya tekhnologiya niobiya i tantala [Chemical technology of niobium and tantalum]. Tomsk, Tomsk Polytechnic University Publ. house, 2010. 97 p.

7. Zelikman A.N., Korshunov B.G., Elyutin A.V. Nioby i tantal [Niobium and tantalum]. Moscow, Metallurgiay Publ., 1990. $295 \mathrm{p}$.

8. Samsonov G.V. Nitridy [Nitrides]. Kiev, Naukova dumka Publ., 1969. $390 \mathrm{p}$

9. Laurjan V.É., Borovinskaya I.P. 0 gorenii alyuminiya v azote [On aluminum combustion in nitrogen]. Physics of combustion and explosion, 2003, vol. 39, no. 5, pp. 45-54.

10. Fizika $i$ khimiya goreniya nanoporoshkov metallov $v$ azotsoderzhashchikh gazovykh sredakh [Physics and Chemistry of Combustion of Metal Nanopowders in Nitrogen-Containing Gas Media]. Ed. by A.A. Gromov. Tomsk, Tomsk University Publ. house, 2007. $322 \mathrm{p}$.

11. Shevchenko V.G., Kononenko V.I., Bulatov M.A. 0 mekhanizme okisleniya poroshkoobraznykh metallov v protsesse ikh nagrevaniya na vozdukhe [On the mechanism of oxidation of powdered metals at their heating in air]. Physics of Combustion and Explosion, 1998, no. 1, pp. 45-49.

12. Khimiya sinteza szhiganiem [Chemistry of synthesis by burning]. Ed. by M. Koizumi. Translated from Japanese. Moscow, Mir Publ., 1998. $247 \mathrm{p}$.

13. Nico C., Monteiro T., Graça M.P.F. Niobium oxides and niobates physical properties: Review and prospects. Progress in Materials Science, 2016, vol. 80, pp. 1-37.

14. Zakorzhevsky V.V. Aluminum Nitride. Concise Encyclopedia of Self-Propagatin High-Temperature Synthesis History, Theory, Technology, and Products. Elsevier, 2017. pp. 16-18.

15. Laassiri S., Zeinalipour-Yazdi C.D., Catlow C.R.A., Hargreaves J.S.J. Nitrogen transfer properties in tantalum nitride based materials. Catalysis Today, 15 May 2017, vol. 286, pp. 147-154.
16. Kyungju Nam, Kicheol Hong, Hyeji Park, Heeman Choe. Facile synthesis of powder-based processing of porous aluminum nitride. Journal of the European Ceramic Society, April 2018, vol. 38, Iss. 4, pp. 1164-1169.

17. Ognjanovic S.M., Winterer M. Optimizing particle characteristics of nanocrystalline aluminum nitride. Powder Technology, 15 February 2018, vol. 326, pp. 488-497.

18. Yamane H., DiSalvo F.J. Sodium flux synthesis of nitrides. Progress in Solid State Chemistry, September 2018, vol. 51, pp. $27-40$.

19. Sahni K., Ashuri M., Emani S., Kaduk J.A., Németh K., Shaw L.L. On the synthesis of lithium boron nitride $\left(\mathrm{Li}_{3} \mathrm{BN}_{2}\right)$. Ceramics International, May 2018, vol. 44, Iss. 7, pp. 7734-7740.

20. Wassner M., Eckardt M., Gebauer C., Bourret G.R., Hüsing N., Behm R.J. Synthesis and electrocatalytic performance of spherical core-shell tantalum (oxy) nitride nitrided carbon composites in the oxygen reduction reaction. Electrochimica Acta, 10 February 2017, vol. 227, pp. 367-381.

21. Fruhstorfer J., Kerber F., Weigelt Ch., Moritz K., Aneziris Ch.G. Activated reaction synthesis of silicon oxynitride from silica and silicon nitride. Ceramics International, May 2018, vol. 44, Iss. 7, pp. 8467-8475.

22. Jinming Jiang, Song Wang, Wei Li, Zhaohui Chen. Low-temperature synthesis of tantalum carbide by facile one-pot reaction. $\mathrm{Ce}$ ramics International, 2016, vol. 42, no. 6, pp. 7118-7124.

23. Gromov A.A., Khabas T.A., Il'in A.P. Goreniye nanoporoshkov metallov [Metal nanopowder combustion]. Ed. by A.A. Gromov. Tomsk, Deltoplan Publ., 2008. 382 p

24. Schweiter C., Schmidt R. Physical Mechanisms of Generation and Deactivation of Singlet Oxygen. Chemical Review, 2003, vol. 103 (5), pp. $1685-1787$.

Received: 18 June 2018.

\section{Information about the authors}

Alexandra O. Chudinova, postgraduate student, National Research Tomsk Polytechnic University.

Alexander P. Ilyin, Dr. Sc., professor, National Research Tomsk Polytechnic University.

Lyudmila O. Root, Cand. Sc., associate professor, National Research Tomsk Polytechnic University.

Andrey V. Mostovshchikov, Cand. Sc., senior researcher, National Research Tomsk Polytechnic University.

Ekaterina A. Bespalova, student, Biysk technological Institute (branch) I.I. Polzunov Altai State Technical University.

Atuiya Manuraj, student, Indian Institute of Technology. 